\title{
Operative intervention for a central pulmonary artery pseudoaneurysm
}

\author{
Abhinav Singh ${ }^{1}$, Yihan Lin ${ }^{1}$, and Jay $\mathrm{Pal}^{2}$ \\ ${ }^{1}$ Affiliation not available \\ ${ }^{2}$ University of Colorado Denver - Anschutz Medical Campus
}

September 24, 2021

\begin{abstract}
Pulmonary artery pseudoaneurysms are a rare but potentially lethal diagnosis. They can be further categorized by etiology or location and are typically successfully treated with endovascular therapies. However, they occasionally require operative intervention. Here, we present a case of a patient who presented with a central pulmonary artery pseudoaneurysm on CT scan with unclear etiology that was initially treated with conservative management. However, this was noted to have rapid enlargement on interval imaging necessitating urgent surgical intervention. The patient underwent a median sternotomy, anterior pulmonary artery arteriotomy for exposure, exclusion of the posterior artery pseudoaneurysm with a bovine pericardial patch, and closure of the anterior arteriotomy with a bovine pericardial patch. The patient did well and was discharged on postoperative day eleven with repeat imaging showing resolution.
\end{abstract}

Title

Operative intervention for a central pulmonary artery pseudoaneurysm

Authors :

Abhinav B. Singh $\mathrm{MD}^{1}$, Yihan Lin MD $\mathrm{MPH}^{2}$, Jay Pal MD PhD ${ }^{2}$

${ }^{1}$ Surgical Outcomes and Applied Research Program, Department of Surgery, University of Colorado, Aurora, Colorado

${ }^{2}$ Division of Cardiothoracic Surgery, Department of Surgery, University of Colorado, Aurora, CO, USA

Corresponding author:

Abhinav B Singh, Abhinav.Singh@cuanschutz.edu

12631 E 17th Ave.

C-305 Aurora, CO 80045

Disclosures: The authors reported no conflicts of interest or funding.

Authors Contributing: AS, YL, and JP all contributed equally to the writing of this case report

Data Availability: The data that support the findings of this study are available on request from the corresponding author. The data are not publicly available due to privacy or ethical restrictions.

Funding: None

IRB: This study is exempt under the institutional review board at our institution. 
Informed Consent : Informed consent was waived as this was exempt under IRB and all patient information has been de identified.

\begin{abstract}
:
Pulmonary artery pseudoaneurysms are a rare but potentially lethal diagnosis. They can be further categorized by etiology or location and are typically successfully treated with endovascular therapies. However, they occasionally require operative intervention. Here, we present a case of a patient who presented with a central pulmonary artery pseudoaneurysm on CT scan with unclear etiology that was initially treated with conservative management. However, this was noted to have rapid enlargement on interval imaging necessitating urgent surgical intervention. The patient underwent a median sternotomy, anterior pulmonary artery arteriotomy for exposure, exclusion of the posterior artery pseudoaneurysm with a bovine pericardial patch, and closure of the anterior arteriotomy with a bovine pericardial patch. The patient did well and was discharged on postoperative day eleven with repeat imaging showing resolution.
\end{abstract}

\title{
Introduction
}

Pulmonary artery pseudoaneurysms (PAPs) are a rare but potentially life-threatening disease ${ }^{1}$. PAPs can be defined as congenital, such as inherited connective tissue abnormalities, or acquired, such as infectious causes $^{1,2,3}$. Clinical presentation ranges from asymptomatic cases to massive hemoptysis with hemorrhagic shock $^{1,2,3}$. PAPs can be characterized as central or peripheral on CT angiography (CTA). There are currently no consensus guidelines for the management of PAPs ${ }^{4}$. Most PAPs are amenable to endovascular treatment with embolization or stenting; however, indications for operative repair include large size, hemorrhage, or medically refractory infections. These usually require an aneurysm resection and often an associated pulmonary resection ${ }^{2,4,5}$. Here we present a case of central PAP with unclear etiology that was treated with median sternotomy and exclusion of the pseudoaneurysm with a pericardial patch.

\section{Case report}

A 52-year-old female presented with chest pain, cough, and shortness of breath over several months. The patient had been evaluated on multiple occasions with an unrevealing work up including an CTA that was normal. However, due to persistent symptoms, a CTA was repeated several weeks later which identified a mediastinal fluid collection and an esophagram showing a contained mid-thoracic esophageal leak. A multidisciplinary team including cardiothoracic surgery, pulmonology, and interventional radiology was convened. Initially, an esophageal stent was considered. However, given the patient's clinical improvement, the patient was medically managed, and a repeat CTA and esophagram showed a decreased size of mediastinal collection and resolution of the esophageal leak. She was therefore discharged on oral antibiotics.

She represented five days later with worsening symptoms. A CTA showed an increase in the size of the mediastinal collection with mass effect on the adjacent right mainstem bronchus, as well as a new $8 \times 5$ $\mathrm{mm}$ right sided central posterior wall PAP (Figure 1). The same multidisciplinary team recommended further work up for malignancy given the enlarging mass despite antibiotic therapy. This consisted of an esophagogastroduodenoscopy and bronchoscopy with ultrasound. Tissue samples obtained were non diagnostic and final pathology demonstrated no malignant cells. An interval CTA, performed two days later to monitor the PAP, demonstrated a significant increase in size to $19 \times 28 \mathrm{~mm}$ (Figure 1) .

Given the enlarging PAP and concern for rupture, it was determined that the patient needed urgent surgical therapy. She underwent a median sternotomy, was placed on cardiopulmonary bypass, and the right pulmonary artery (PA) was exposed. The posterior right PA artery was encased in a dense mass, which was biopsied. Due to the inflammatory process, we elected to repair the pseudoaneurysm from within the pulmonary artery rather than resecting and replacing the involved vessel. A longitudinal anterior right pulmonary arteriotomy was performed with a $1.5 \mathrm{~cm}$ perforation noted in the posterior wall(Figure 2). A bovine pericardial patch was sutured around the borders of the PAP, effectively excluding it from the main PA lumen(Figure 2). The anterior arteriotomy was closed with another bovine pericardial patch to avoid stenosis. Multiple intraoperative tissues samples were sent to evaluate for malignancy with non-diagnostic 
results from pathology. The patient was weaned off cardiopulmonary bypass, for a total time of 126 minutes, and the sternum was closed.

The patient's post-operative course was uneventful. She was extubated on postoperative day one and discharged on post-operative day eleven on intravenous antibiotics. The patient's symptoms have since resolved since discharge, with interval imaging showing resolution of the right PAP as well as the mediastinal collection.

\section{Discussion}

In this case we present a unique presentation and management strategy for a PAP. The patient had imaging studies that showed an esophageal leak and a mediastinal collection with subsequent mass effect and new PAP concerning for a neoplastic versus infectious process. All attempts to elucidate the etiology were unsuccessful as pathological and microbiological data were unrevealing. Given the unique finding of a central PAP with rapid expansion, urgent operative surgical intervention was performed because of the risk of rupture. Intraoperatively, given the posterior location of PAP and adjacent mediastinal mass, a right anterior pulmonary arteriotomy provided optimal exposure (Figure 2) . The PAP was excluded with bovine pericardial patch, which lead resolution of the PAP on post-operative CTA. No pulmonary resection was required. Finally, the patient's PAP was likely caused by an infection, as the mediastinal collection resolved after antimicrobial therapy; however, the causative organism as well as the cause for esophageal perforation was never identified.

\section{Conclusions}

Central pulmonary artery pseudoaneurysms are rare and can be safely treated with pulmonary artery arteriotomy and bovine patch exclusion.

\section{Conflict of interests}

The authors declare that there are no conflict of interests.

\section{References}

1. Chen Y, Gilman M, Humphrey K, Salazar G, Sharma A, Muniappan A, et al. Pulmonary Artery Pseudoaneurysms: Clinical Features and CT Findings. AJR Am J Roentgenol . 2017;208:84-91

2. Guillaume B, Vendrell A, Stefanovic X, Thony F, Ferretti G. Acquired Pulmonary Artery Pseudoaneurysms: a Pictorial Review. Br J Radiol . 2017;90:20160783-20160783

3. Kaufman J. Pulmonary Circulation: In: Kaufman J, Lee M, eds. Vascular and Interventional Radiology the Requisites . $2^{\text {nd }}$ ed. Philadelphia. 2014: 159-176

4. Kalra-Lall A, Donaldson, J Martin CE $3^{\text {rd }}$. Brief Review: Pulmonary Artery Aneurysms and Pseudoaneurysms. Int J Cardiovasc Imaging . 2019;35:1357-1364

5. Theodoropoulos P, Ziganshin B, Tranquilli M, Elefteriades J. Pulmonary Artery Aneurysms: Four Case Reports and Literature Review. Int J Angiol . 2013;22:143-148

\section{Hosted file}

JCS- Case report PA PSA Figures.docx available at https://authorea.com/users/434953/articles/ 538308-operative-intervention-for-a-central-pulmonary-artery-pseudoaneurysm 